\title{
A JITTER DETECTION METHOD BASED ON THE INTEGRATION IMAGING MODEL
}

\author{
G. Ye ${ }^{1}$, J. Pan ${ }^{1, *}$, Y. Zhu ${ }^{1}$, S. Jin ${ }^{1}$ \\ ${ }^{1}$ State Key Laboratory of Information Engineering in Surveying, Mapping and Remote Sensing, Wuhan University, Wuhan 430079, \\ China - (yeguo69, panjun1215, yzhu1003,jsy)@whu.edu.cn
}

\section{Commission III, ICWG III/IVb}

KEY WORDS: Jitter detection, Multispectral images, Parallax map, Imaging process, ZY-3 satellite

\begin{abstract}
:
Satellite jitter is a random error source which leads to image degradation. This paper proposes a method to detect the time-variant jitter using multispectral images. In the method, multispectral images are adopted for their large overlap to obtain the parallax map. The imaging process is analyzed in details, and an integration imaging model is constructed, which takes fully into account the timevariant jitter property and builds the relationship between object space with image space. Besides, multispectral images of ZY-3 satellite were used for experiments, and results show that the presented method obtains the jitter curve with the error of amplitude, frequency and phase not more than $0.0591 \mathrm{px}, 0.0006 \mathrm{~Hz}$ and $0.007 \mathrm{rad}$, respectively. Results demonstrate the performance of the presented method in jitter detection.
\end{abstract}

\section{INTRODUCTION}

\subsection{Satellite jitter}

With the development of remote sensing technology, the resolution of optical sensors has been greatly improved, and high-resolution satellites have been widely used in mapping, monitoring and other fields. However, satellite jitter will lead to image degradation, which severely limits the application of high-precision remote sensing images (Mattson et al., 2010; Tong et al., 2015a).

\begin{tabular}{|l|c|c|c|}
\hline $\begin{array}{l}\text { Satellite } \\
\text { /Sensor }\end{array}$ & $\begin{array}{c}\text { Launch } \\
\text { Time }\end{array}$ & Country & Jitter \\
\hline ASTER & 1999 & Japan & $0.2 \mathrm{px}, 1.5 \mathrm{~Hz}$ \\
\hline QuickBird & 2001 & USA & $\begin{array}{c}5 \mathrm{px}, 1 \mathrm{~Hz} ; \\
0.2 \mathrm{px}, 4.3 \mathrm{~Hz}\end{array}$ \\
\hline ALSat-1 & 2002 & Algeria & $0.5 \mathrm{~Hz}$ \\
\hline MEX-HRSC & 2003 & Germany & $\begin{array}{c}0.1-0.2 \mathrm{~Hz} \\
1.7 \mathrm{~Hz}\end{array}$ \\
\hline UK-DMC & 2003 & UK & $0.6 \mathrm{~Hz}$ \\
\hline ALOS & 2006 & Japan & $\begin{array}{c}1 \mathrm{~km}, 6-7 \mathrm{~Hz} ; \\
100 \mathrm{~m}, 60-70 \mathrm{~Hz}\end{array}$ \\
\hline $\begin{array}{l}\text { Mapping } \\
\text { satellite-1 }\end{array}$ & 2010 & China & $\begin{array}{c}0.1 \mathrm{px}, 0.105 \mathrm{~Hz} ; \\
0.05 \mathrm{px}, 0.635 \mathrm{~Hz} ; \\
0.05 \mathrm{px}, 4 \mathrm{~Hz}\end{array}$ \\
\hline Pleiades-HR & 2011 & France & $70.9-78.4 \mathrm{~Hz}$ \\
\hline $\begin{array}{l}\text { Gaofen-1 } \\
\text { 02/03/04 } \\
\text { satellites }\end{array}$ & 2018 & China & $\begin{array}{c}0.077-1.3 \mathrm{px}, \\
1.1-1.2 \mathrm{~Hz}\end{array}$ \\
\hline
\end{tabular}

Table 1. Examples of satellite jitter

Satellite jitter is a random error source due to the periodic motion of the the dynamic structure on board, considerable temperature variation caused by orbital changes, and so on (Iwasaki et al., 2011; Tong et al., 2014). In the push-broom imaging process of optical satellites, satellite jitter directly leads to image distortion and blur, reduces image geometric and radiometric quality, and then affects the application of multisource image fusion, change detection, and other subsequent high-precision remote sensing operations (Wang et al., 2016; Hu et al., 2018). Jitter has been found on many satellites, such as ASTER (Teshima and Iwasaki, 2008), QuickBird (Ayoub et al., 2008), ALSat-1 (Mumtaz and Palmer, 2013), MEX-HRSC (Gwinner et al., 2013), UK-DMC (Mumtaz and Palmer, 2013), ALOS (Takaku and Tadono, 2010), Mapping satellite-1 (Sun et al., 2015), Pleiades-HR (Amberg et al., 2013), Gaofen-1 02/03/04 satellites (Zhu et al., 2018) and so on, as listed in Table 1 .

The image degradation caused by satellite jitter will be even worse, if the satellites use the TDI CCD (time delay integration charge coupled devices) imaging system (Wang et al., 2012). TDI CCD is widely used in high-resolution satellites, because it can improve sensitivity and signal-to-noise ratio of the camera. It's the integration characteristic that gives TDI CCD the advantage, but it also makes TDI CCD sensitive to satellite jitter (Chamberlain and Washkurak, 1990; Wong et al., 1992; Fan and Zhang, 2010). Therefore, it's necessary to do some investigate on the satellite jitter with TDI CCD on board.

\subsection{Jitter detection}

In order to eliminate the negative effect of satellite jitter on the quality of the images and their products, jitter detection is one of key steps in ground processing systems.

There are two main approaches to achieve the jitter detection. One is to directly take the measurements using the highprecision attitude sensor, such as angular velocity transducer, angular displacement transducer, etc. For example, the angular displacement sensor (ADS) was adopted to detect highfrequency jitter in Landsat (Barker and Seiferth, 1996). Panchromatic Remote-sensing Instrument for Stereo Mapping (PRISM) was used for attitude fluctuation observation of ALOS (Takaku and Tadono, 2010). The other is to obtain the regularity of jitter change indirectly through the images. Teshima and Iwasaki (2008) achieved jitter detection of Terra Spacecraft using ASTER/SWIR imagery. Sun et al. (2015) detected satellite jitter based on short-time asynchronous images. Tong et al. (2014) proposed three methods respectively using multispectral images, stereo images and panchromatic images 
for jitter detection. Generally, the former direct approach relies on the accuracy of the attitude sensor and has higher requirements on the hardware, which limits its application. Whereas the latter one is more economic and efficient, so that it has been more widely adopted in studies (Teshima and Iwasaki, 2008; Tong et al., 2015b; Wang et al., 2017).

Although there are many researches on jitter detection, the accuracy of the obtained results still can be improved. In this paper, an integration imaging model is proposed and a method based on it is conducted. The main contribution is the transformation from disparity curve to jitter curve provided by the integration imaging model. Main steps of the presented method will be illustrated following the introduction and experiments will be undertaken to validate the presented method.

\section{METHODOLOGY}

This paper proposes an integration imaging model to describe the time-variant jitter, and then a method based on it is adopted for jitter detection with multispectral images used. Fig.1 shows the workflow of the proposed method. First, the pixel-to-pixel matching is conducted to the large overlap between multispectral images to obtain the parallax map. Then, according to the parallax map, parallax disparities averaged by line are calculated, and the disparity curve is fitted. Finally the integration imaging model is employed to obtain the jitter curve.

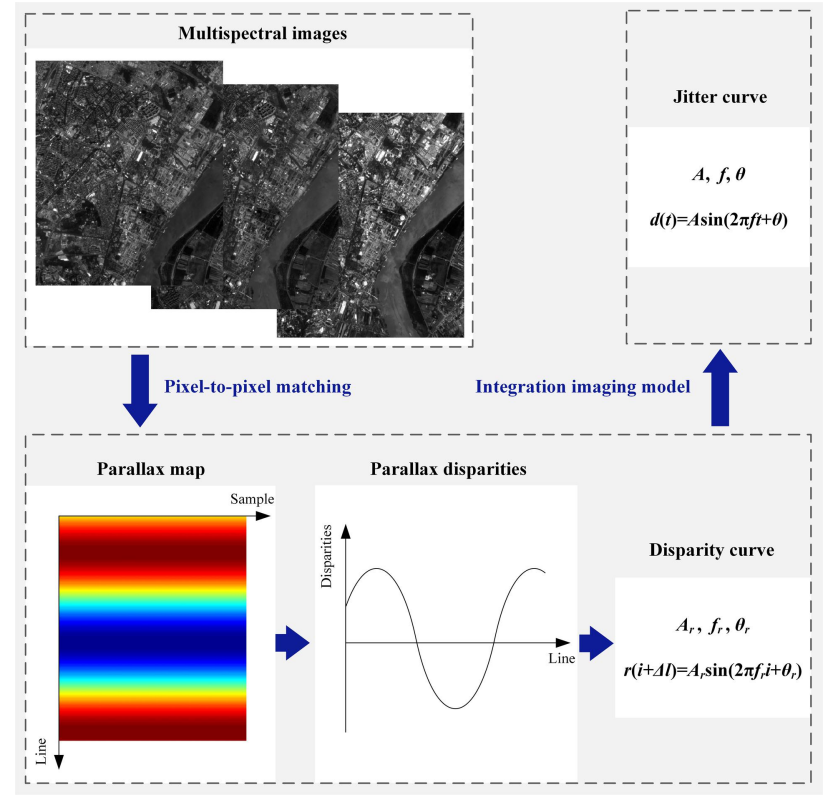

Figure 1. Overview of the presented method

\subsection{Pixel-to-pixel matching}

There are many bands in multispectral images, where blue, green, red and infrared bands are arranged in parallel on the camera focal plane at certain intervals. Since the spectral characteristic of the infrared band varies from that of visible bands, visible bands, i.e. blue (B1), green (B2) and red (B3), are adopted for good performance of image matching in jitter detection. In order to obtain high matching accuracy, adjacent visible bands are used, that is the combination of $\mathrm{B} 1$ and $\mathrm{B} 2$, or B2 and B3. Fig. 2 shows the adopted image bands and camera focal plane, where $l_{1}$ and $l_{2}$ are the distance between $\mathrm{B} 1$ and B2 bands, B2 and B3 bands, respectively, the overlap area of three bands are remarked in the orange box. The imaging intervals between different image bands, which are placed in parallel on the camera focal plane, correspond to the distances between them. Because the distance between adjacent bands is fixed, if the coarse matching is employed on their overlap area, the accuracy of the obtained initial pixels will meet the pixellevel requirement. After that, a higher precision can be achieved by further matching, according to the obtained pixels (Tong et al., 2015b; Zhu et al., 2018). Thus, the pixel-to-pixel matching is done through coarse matching followed by fine matching.

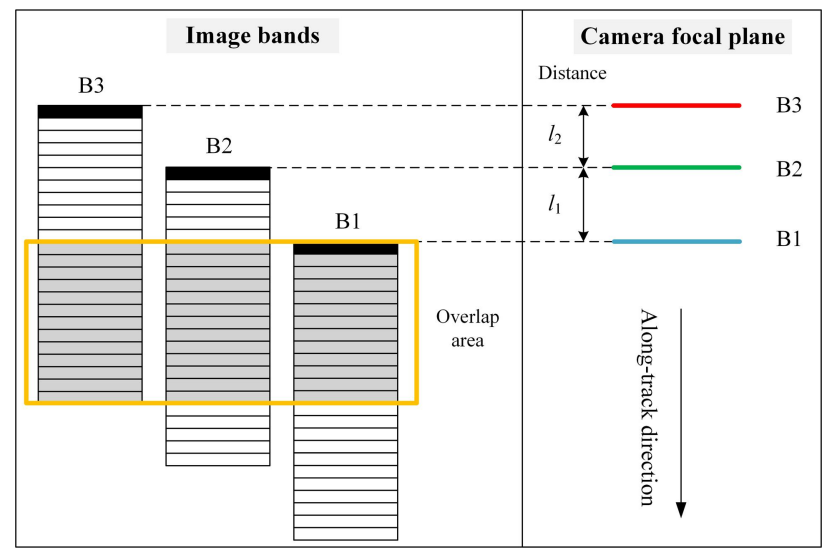

Figure 2. Image bands and camera focal plane

Image matching is employed on corresponding lines between adjacent bands, and correlation coefficient is used for coarse matching, least squares algorithm is utilized for fine matching. First, the initial corresponding point is obtained in the neighborhood of the initial image point. The correlation coefficient is used as a measurement tool of coarse matching: the point with the maximum correlation coefficient is selected as the initial corresponding point. The correlation coefficient $\rho(c, r)$ is calculated as follows (Potucková, 2004):

$$
\rho(c, r)=\frac{\sum_{i=1}^{m} \sum_{j=1}^{n}\left(g_{i, j} \cdot g_{i+r, j+c}^{\prime}\right)-\frac{1}{m n}\left(\sum_{i=1}^{m} \sum_{j=1}^{n} g_{i, j}\right)\left(\sum_{i=1}^{m} \sum_{j=1}^{n} g_{i+r, j+c}^{\prime}\right)}{\sqrt{\left[\sum_{i=1}^{m} \sum_{j=1}^{n}\left(g_{i, j}\right)^{2}-\frac{1}{m n}\left(\sum_{i=1}^{m} \sum_{j=1}^{n} g_{i, j}\right)^{2}\right]\left[\sum_{i=1}^{m} \sum_{j=1}^{n}\left(g_{i+r, j+c}^{\prime}\right)^{2}-\frac{1}{m n}\left(\sum_{i=1}^{m} \sum_{j=1}^{n} g_{i+r, j+c}^{\prime}\right)^{2}\right]}}
$$

where $(r, c)$ is the coordinate difference between the target point and candidate point, $m$ and $n$ are the size of the target window, $i$ and $j$ are the line number and sample number in the target window respectively, $g_{i, j}$ and $g_{i+, j+c}^{\prime}$ are the DN values respectively in the target window and the search window.

Afterwards, the least square matching algorithm is adopted to obtain the sub-pixel matching results. According to the error observation model, the geometric and radiometric mapping relation between target window and research window is calculated, so that the accurate corresponding point is determined. The observation model is expressed as follows (Bethel, 1997; Potucková, 2004):

$g_{1}(x, y)+n_{1}(x, y)=h_{0}+h_{1} \cdot g_{2}\left(a_{0}+a_{1} x+a_{2} y, b_{0}+b_{1} x+b_{2} y\right)+n_{2}(x, y)$

where $(x, y)$ is the coordinate of the target point; $a_{0}, a_{1}, a_{2}, b_{0}$, $b_{0}, b_{1}, b_{2}$ are respectively the parameter of the geometric affine transformation; $h_{0}$ and $h_{1}$ are the parameters of the gray transformation; $g_{1}(*)$ and $g_{2}(*)$ are respectively the DN 
values corresponding to the left and right matching windows; $n_{1}(*)$ and $n_{2}(*)$ are the noise respectively corresponding to the left and right matching windows. The transformation parameters in the formula are calculated by the least square matching algorithm, and then the accurate corresponding points are obtained through the geometric affine transformation.

The parallax map records the coordinate differences between the corresponding points, and parallax disparities averaged by line are calculated from that. A sine function is used to fit the parallax disparities, and then image displacement can be obtained.

\subsection{Integration imaging model}

The disparity in parallax maps represents the overall effect, and it cannot reflect the real-time changes of satellite jitter. The integration imaging model is built to create a connection between parallax disparities in disparity curve and jitter offset in jitter curve, with image displacement as the bridge, as shown in Fig.3.

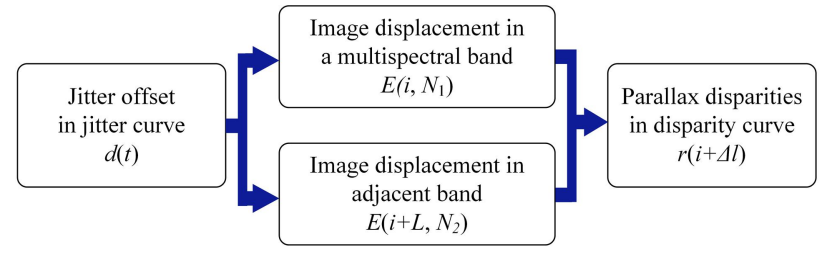

Figure 3. Workflow of the integration imaging model

TDI CCDs (time delay integration charge coupled devices) is the natural component in remote imaging sensing system, and its imaging process is accompanied by charge transfer and accumulation (Chamberlain and Washkurak, 1990; Hochman et al., 2004). Fig. 4 shows the schematic of TDI CCD, where $M$ is the pixel number in across-track direction and $N$ is the integration stages of TDI CCD (Wong et al., 1992). Each stage of TDI CCD scan the same ground area in turn, and charge is transferred and accumulated in synchrony with the optically scanned image. Until $N$ integration periods are completed, all generated charges are transferred to the register, and the image line is read out. TDI CCD has the advantage of increasing total amount of light captured, however, jitter offset will mismatch between the CCD motion speed and charge transfer rate, which leads to the image displacement.

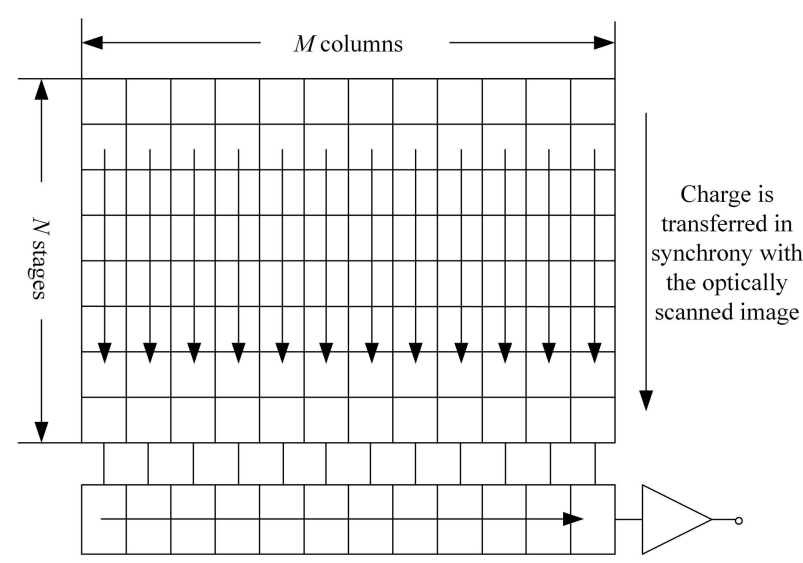

Figure 4. Schematic of TDI CCD (Wong et al., 1992)
The jitter offset at imaging time $t$, i.e. $d(t)$, can be described by the sine function (Hadar et al., 1992), that is

$$
d(t)=A \sin (2 \pi f t+\theta)
$$

where $A$ is the amplitude, $f$ is the frequency and $\theta$ is the initial phase. The jitter offsets are accumulated during the TDI CCD integration imaging, and they are transmitted to the image plane, resulting the image displacement. Take $N$ integration stages of TDI CCD for instance, $i$ is recorded as the line number, $T$ is recorded as the time needed for one integration, the image displacement $E(i, N)$ in a multi-spectral band can be given by

$$
\begin{aligned}
E(i, N)= & \frac{1}{N}\left[\frac{1}{2} d((i-N) T)+\sum_{k=1}^{N-1} d((i-N+k) T)+\frac{1}{2} d(i T)\right] \\
= & \frac{1}{N}\left[\frac{1}{2} A \sin (2 \pi f T i-2 \pi f T N+\theta)\right. \\
& \left.+A \sum_{k=1}^{N-1} \sin (2 \pi f T i+2 \pi f T(k-N)+\theta)+\frac{1}{2} A \sin (2 \pi f T i+\theta)\right]
\end{aligned}
$$

where

$$
\begin{aligned}
& \sum_{k=1}^{N-1} \sin (2 \pi f T i+2 \pi f T(k-N)+\theta) \\
= & \sin (2 \pi f T i+2 \pi f T(1-N)+\theta)+\sin (2 \pi f T i+2 \pi f T(-1)+\theta) \\
& +\sin (2 \pi f T i+2 \pi f T(2-N)+\theta)+\sin (2 \pi f T i+2 \pi f T(-2)+\theta) \\
& +\cdots+\sin \left(2 \pi f T i+2 \pi f T\left(\frac{N}{2} N\right)+\theta\right) \\
= & 2 \cos (\pi f T(2-N)) \sin (2 \pi f T i-\pi f T N+\theta) \\
& +\cdots+\sin (2 \pi f T i-\pi f T N+\theta) \\
= & {\left[\sum_{k=1}^{\frac{N}{2}-1} 2 \cos (\pi f T(2 k-N))+1\right] \sin (2 \pi f T i-\pi f T N+\theta) }
\end{aligned}
$$

Substituting Eq. (5) into Eq. (4), there is

$$
\begin{aligned}
E(i, N)= & \frac{A}{N}\{\cos (\pi f T N) \sin (2 \pi f T i-\pi f T N+\theta) \\
& \left.+\left[\sum_{k=1}^{\frac{N}{2}-1} 2 \cos (\pi T f(2 k-N))+1\right] \sin (2 \pi f T i-\pi f T N+\theta)\right\} \\
= & \frac{A}{N}\left[\cos (\pi f T N)+\sum_{k=1}^{\frac{N}{2}-1} 2 \cos (\pi f T(2 k-N))+1\right] \sin (2 \pi f T i-\pi f T N+\theta)
\end{aligned}
$$

Since the parallax disparity $r(i)$ reflects the difference between image displacements of corresponding lines in adjacent bands, i.e. $E\left(i+L, N_{2}\right)$ and $E\left(i, N_{1}\right)$, the relationship can be expressed as

$$
r(i)=E\left(i+L, N_{2}\right)-E\left(i, N_{1}\right)
$$

where $i$ is the line number, $L$ is the distance between the two bands, $N_{1}$ and $N_{2}$ are the integration stages of TDI CCD for adjacent bands respectively. By substituting Eq. (6) into Eq. (7), the parallax disparity can be calculated by 


$$
\begin{aligned}
r(i)= & \frac{A}{N_{2}}\left[\cos \left(\pi f T N_{2}\right)+\sum_{k=1}^{\frac{N_{2}}{2}-1} 2 \cos \left(\pi f T\left(2 k-N_{2}\right)\right)+1\right] \sin \left(2 \pi f T(i+L)-\pi f T N_{2}+\theta\right) \\
& -\frac{A}{N_{1}}\left[\cos \left(\pi f T N_{1}\right)+\sum_{k=1}^{\frac{N_{1}-1}{2}} 2 \cos \left(\pi f T\left(2 k-N_{1}\right)\right)+1\right] \sin \left(2 \pi f T i-\pi f T N_{1}+\theta\right)
\end{aligned}
$$

In order to simplify the expression, $C, \alpha, D$ and $\beta$ are used as the substitutes.

$$
\left\{\begin{array}{l}
C=\frac{1}{N_{2}}\left[\cos \left(\pi f T N_{2}\right)+\sum_{k=1}^{\frac{N_{2}}{2}-1} 2 \cos \left(\pi f T\left(2 k-N_{2}\right)\right)+1\right] \\
\alpha=2 \pi f T L-\pi f T N_{2}+\theta \\
D=-\frac{1}{N_{1}}\left[\cos \left(\pi f T N_{1}\right)+\sum_{k=1}^{\frac{N_{1}}{2}-1} 2 \cos \left(\pi f T\left(2 k-N_{1}\right)\right)+1\right] \\
\beta=-\pi f T N_{1}+\theta
\end{array}\right.
$$

Thus, Eq. (8) can expressed as:

$$
\begin{aligned}
r(i) & =A[C \sin (2 \pi f T i+\alpha)+D \sin (2 \pi f T i+\beta)] \\
& =A\left[\sqrt{C^{2}+D^{2}+2 C D \cos (\alpha-\beta)} \sin \left(2 \pi f T i+\frac{\alpha+\beta}{2}+\phi\right)\right]
\end{aligned}
$$

where

$$
\phi=\arctan \left(\frac{C-D}{C+D} \tan \frac{\alpha-\beta}{2}\right)
$$

It worth noting that because the model works on the overlap of the three bands, while the overlap of B2 and B3 bands is larger than that of the three bands, the interval should be taken into account when jitter detection is conducted by using B2 and B3 bands. $r(i+\Delta l)$ is used to replace $r(i)$ to represent parallax disparity between adjacent bands, where $\Delta l=0$ for B1-B2 and $\Delta l=l_{1}$ for $\mathrm{B} 2-\mathrm{B} 3 ; l_{1}$ and $l_{2}$ are the distance between $\mathrm{B} 1$ and $\mathrm{B} 2, \mathrm{~B} 2$ and $\mathrm{B} 3$, respectively. A sine function is used to describe the parallax disparity $r(i+\Delta l)$, i.e.

$$
r(i+\Delta l)=A_{r} \sin \left(2 \pi f_{r} i+\theta_{r}\right)
$$

where the amplitude $A_{r}$, frequency $f_{r}$ and initial phase $\theta_{r}$ are obtained based on Eq. (9) (10) and (11), which can be expressed as follows:

$$
\left\{\begin{aligned}
A_{r}= & A \sqrt{C^{2}+D^{2}+2 C D \cos \left(2 \pi f T L+\pi f T\left(N_{1}-N_{2}\right)\right)} \\
f_{r}= & f T \\
\theta_{r}= & \theta+2 \pi f T \Delta l+\pi f T L-\pi f T\left(\frac{N_{1}+N_{2}}{2}\right) \\
& +\arctan \left(\frac{C-D}{C+D} \tan \left(\pi f T L+\pi f T\left(\frac{N_{1}-N_{2}}{2}\right)\right)\right)
\end{aligned}\right.
$$

Therefore, the relationship between parameters of jitter offset ( $A, f, \theta)$ and parameters of parallax disparity $\left(A_{r}, f_{r}, \theta_{r}\right)$ can be given by

$$
\left\{\begin{array}{l}
A=A_{r} / \sqrt{C^{2}+D^{2}+2 C D \cos \left(2 \pi f T L+\pi f T\left(N_{1}-N_{2}\right)\right)} \\
f=f_{r} / T \\
\theta=\theta_{r}-2 \pi f_{r} \Delta l-\pi f T L+\pi f T\left(\frac{N_{1}+N_{2}}{2}\right) \\
\quad-\arctan \left(\frac{C-D}{C+D} \tan \left(\pi f T L+\pi f T\left(\frac{N_{1}-N_{2}}{2}\right)\right)\right)
\end{array}\right.
$$

where $T$ is the single integration time of TDI CCD, $N_{1}$ and $N_{2}$ are the integration stages of TDI CCD for adjacent bands respectively, $L$ is the distance between the two bands ( $L=l_{1}$ for B1-B2 and $L=l_{2}$ for B2-B3), interval $\Delta l=0$ for B1-B2 and $\Delta l=l_{1}$ for B2-B3, $C$ and $D$ are expressed in Eq.(9). Especially, if $N_{1}=N_{2}$, there is

$$
\left\{\begin{array}{l}
A=A_{r} N_{1} / 2 \sin (\pi f T L)\left[\cos \left(\pi f T N_{1}\right)+\sum_{k=1}^{\frac{N_{1}}{2}-1} 2 \cos \left(\pi f T\left(2 k-N_{1}\right)\right)+1\right] \\
f=f_{r} / T \\
\theta=\theta_{r}-2 \pi f_{r} \Delta l-\pi f T L+\pi f T N_{1}-\frac{\pi}{2}
\end{array}\right.
$$

The integration imaging model achieves the transformation from parallax disparities to jitter offset, in fact, it also connects the image space and object space so as to make the detailed time-variant jitter extracted from the overall effect in images.

\section{EXPERIMENTS AND ANALYSIS}

\subsection{Data description}

The performance of the presented method was checked using a number of multispectral images of ZY-3 satellite.

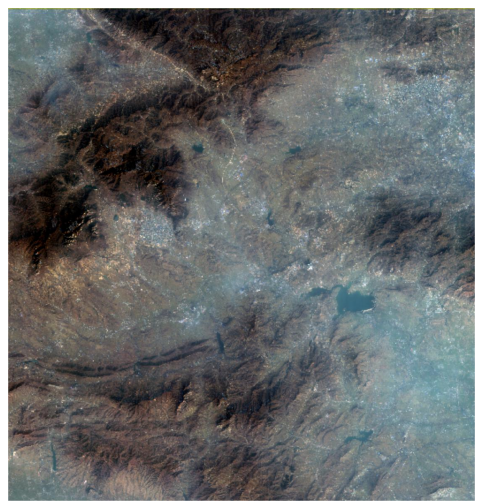

Figure 5. Multispectral image of ZY-3 satellite

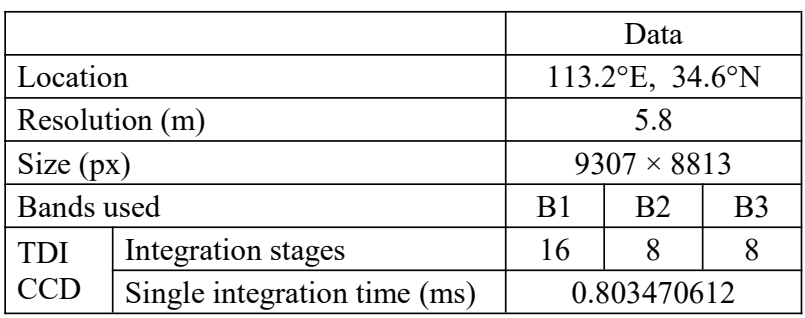

Table 2. Description of the experimental data

ZY-3 satellite is equipped with $5.8-\mathrm{m}$ optical cameras, capable of recording images in four spectral bands: blue (B1), green (B2), red (B3), and infrared (B4). The B1, B2, and B3 bands are 
used for experiments. The adopted data, near Dengfeng, Henan province, China, was originally collected on 3rd February 2012, as shown in Fig. 5. Table 2 lists the detailed description of the experimental data.

\subsection{Results}

Fig. 6 shows the parallax maps of experimental data after pixel to pixel matching, where the results obtained with B1-B2 and B2-B3 bands are respectively demonstrated in Fig. 6(a) and (b). The matching was conducted in both across- and along-track direction. An apparent sinusoidal jitter can be found according to parallax maps.
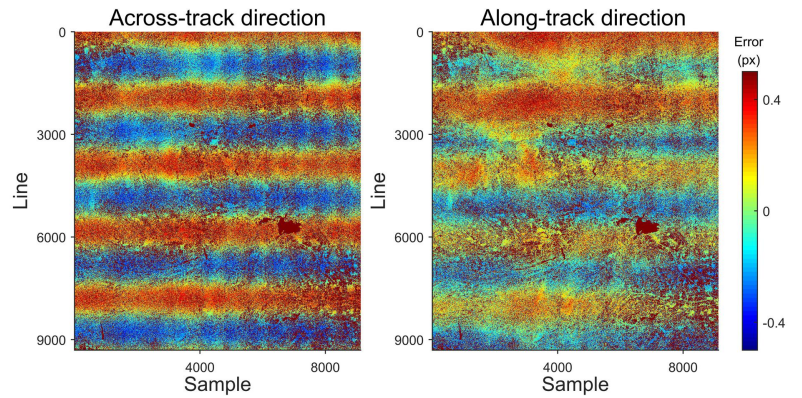

(a)
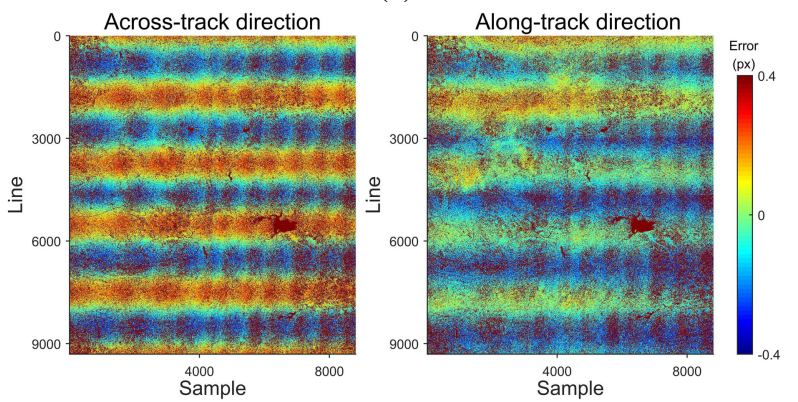

(b)

Figure 6. Parallax maps obtained with (a) B1-B2 used and (b) B2-B3 used
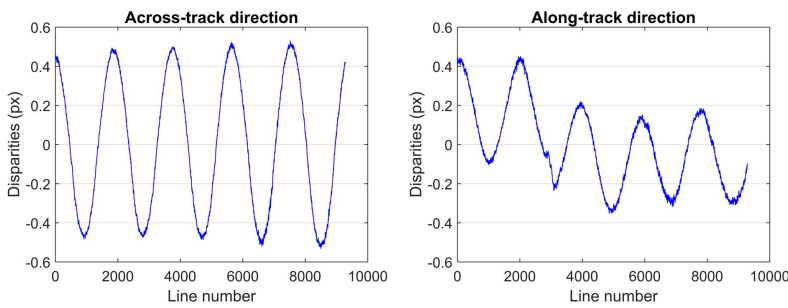

(a)
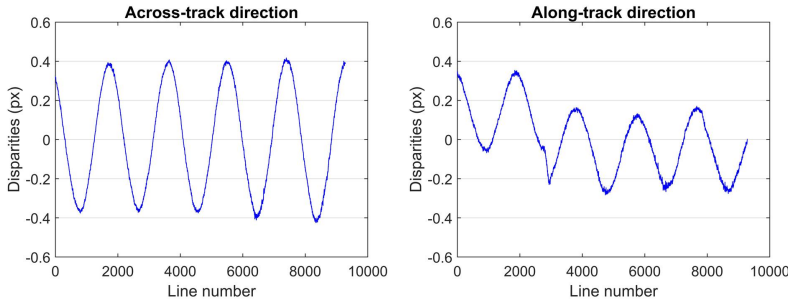

(b)

Figure 7. Parallax disparities between (a) B1 and B2, (b) B2 and $\mathrm{B} 3$, respectively

In addition to the parallax maps, the parallax disparities averaged by line are displayed in Fig. 7. Fig. 7(a) and (b) shows the parallax disparities, respectively related to Fig. 6(a) and (b) with B1-B2 and B2-B3 bands used separately. The distribution of disparities in across-track direction shows regular sine curve, whereas disparities in along-track direction is not agreement with that in across-track direction. Even though disparities in along-track also waves along the image line number, such pattern cannot expressed directly by a sine function. This is because the topographic information of this area covered by the experiment data varies a lot, which makes the disparities composed of not only image motion caused by satellite jitter but also image parallax due to elevation change. Many mountains are in the experiment data which has been displayed in Fig. 5, so that the topographic information affects the jitter detection in the along-track direction because the parallax due to topography may greater than that due to satellite jitter. Thus, in order to extract the offset caused by jitter itself, the experiments were undertaken in across-track direction.

\begin{tabular}{|l|c|c|}
\hline & B1-B2 & B2-B3 \\
\hline Amplitude (px) & 0.4941 & 0.3830 \\
\hline Frequency (Hz) & $5.2764 \times 10^{-4}$ & $5.2756 \times 10^{-4}$ \\
\hline Phase (rad) & 1.6854 & 2.1558 \\
\hline
\end{tabular}

Table 3. Parameters of the disparity curve

The parallax disparities are fitted by the sine function and parameters of the fitted disparity curve are listed in Table 3 . The distances vary between multispectral bands, resulting in different imaging intervals of corresponding lines in each band, so that parallax obtained with different bands is different. Hence, the amplitude or phase obtained by using B1-B2 varies from that obtained with B2-B3 bands, and only the frequency is similar. According to the integration imaging model, the disparity curve is transferred to the jitter curve, as shown in Table 4. The jitter with amplitude, frequency, phase in terms of approximately $0.9 \mathrm{px}, 0.6 \mathrm{~Hz}$ and $-0.1 \mathrm{rad}$ is detected, where the deviation between B1-B2 and B2-B3 is no more than $0.0567 \mathrm{px}$, $0.0001 \mathrm{~Hz}$ and $0.0004 \mathrm{rad}$. The small deviation indicates that the presented method is reliable.

\begin{tabular}{|l|c|c|c|}
\hline & B1-B2 & B2-B3 & deviation \\
\hline Amplitude (px) & 0.9662 & 0.9095 & 0.0567 \\
\hline Frequency (Hz) & 0.6567 & 0.6566 & 0.0001 \\
\hline Phase (rad) & -0.1173 & -0.1177 & 0.0004 \\
\hline
\end{tabular}

Table 4. Parameters of the jitter curve

Due to the high geometric accuracy and ability to reflect the real ground conditions, the 2-m orthoimage is further utilized for providing the reference value of the jitter curve, so as to verify the presented method in this paper. For objectivity, ERDAS IMAGINE, is used for image registration between each band and their corresponding orthoimage. A total of 3737, 3416, 2816 GCPs are obtained for B1, B2, B3 band respectively. Fig.8 shows the registration residuals between each band with the orthoimage.

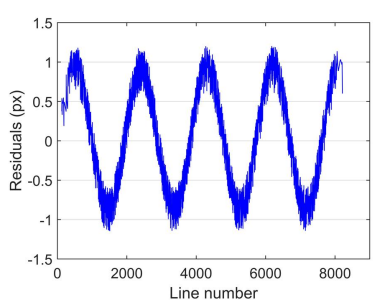

(a)

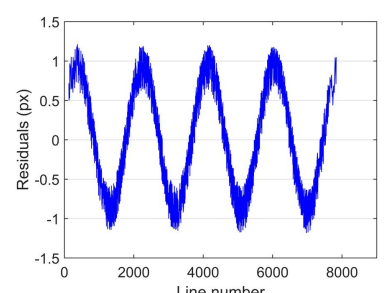

(b) 


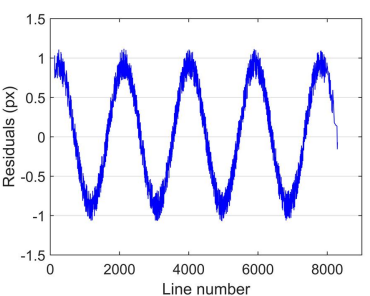

(c)

Figure 8. Registration residuals between the orthoimage and (a) B1, (b) B2, (c) B3, respectively

The registration residuals between the orthoimage and each band is in fact the image motion in the band, which is formulated in Eq. (6). It is worth noting that the overlap area between bands is used, and the time varies when bands scan the corresponding line, so that the intervals between bands should be taken into account. If $l_{1}$ represents the distance between B1 and B2, $l_{2}$ represents the distance between B2 and B3, according to Eq. (6), the parallax between the orthoimage and the band $E_{0}$ can be expressed as:

$$
\begin{aligned}
E_{0}= & E(i+\Delta l, N) \\
= & \frac{A}{N}\left[\cos (\pi f T N)+\sum_{k=1}^{\frac{N}{2}-1} 2 \cos (\pi f T(2 k-N))+1\right] \\
& \cdot \sin (2 \pi f T(i+\Delta l)-\pi f T N+\theta)
\end{aligned}
$$

where $\Delta l=0$ for $\mathrm{B} 1, \Delta l=l_{1}$ for $\mathrm{B} 2, \Delta l=l_{1}+l_{2}$ for $\mathrm{B} 3$, respectively. Thus, the integration imaging model is expended for the use of orthoimage, which can be expressed as

$$
\left\{\begin{array}{l}
A=N A_{r} /\left[\cos \pi T N f+\sum_{k=1}^{\frac{N}{2}-1} 2 \cos (\pi T(2 k-N) f)+1\right] \\
f=f_{r} / T \\
\theta=\theta_{r}-2 \pi f_{r} \Delta l+\pi f T N
\end{array}\right.
$$

Hence, the reference value of the jitter curve can be derived from the registration residuals between each image band and the orthoimage. The detailed values, obtained by using B1, B2 and B3 respectively, are listed in Table 5. As there is the inevitable calculation error, the average value is taken as the final reference of the jitter curve.

\begin{tabular}{|l|c|c|c|c|}
\hline & B1 & B2 & B3 & average \\
\hline Amplitude (px) & 0.9047 & 0.9145 & 0.9022 & 0.9071 \\
\hline Frequency (Hz) & 0.6552 & 0.6573 & 0.6559 & 0.6561 \\
\hline Phase (rad) & -0.1031 & -0.1313 & -0.0976 & -0.1107 \\
\hline
\end{tabular}

Table 5. Reference values of the jitter curve

Table 6 lists the detected results of the jitter curve, including a comparison with the method based on the jitter displacement estimation model (Pan et al., 2017), where the error illustrates the difference between the detected value and the reference value. As shown in Table 6, the error of amplitude, frequency and phase in the jitter curve is not more than $0.0591 \mathrm{px}, 0.0006$ $\mathrm{Hz}$ and $0.007 \mathrm{rad}$, respectively. Besides, the results illustrate that the main differences between the method based on the jitter displacement estimation model and the presented method in this paper are the amplitude and phase. The frequencies obtained by two method are the same. In contrast, this pattern cannot be observed from the amplitude and phase. Anyway, the errors of both amplitude and phase detected by the presented method are smaller than that obtained by the method based on the jitter displacement estimation model. Taking the results obtained by using B1 and B2 bands for instance, for the amplitude, the error in the method based on the jitter displacement estimation model is $0.0840 \mathrm{px}$, while that in the presented method is $0.0591 \mathrm{px}$; for the phase, the error in the method based on the jitter displacement estimation model is $0.0267 \mathrm{rad}$, while that in the presented method is $0.0066 \mathrm{rad}$. It validates the advantage of the integration imaging model, which takes fully into account the time-variant characteristic of satellite jitter in the imaging

\begin{tabular}{|c|c|c|c|c|c|}
\hline & & & $\begin{array}{c}\text { Amplitude } \\
\text { (px) }\end{array}$ & $\begin{array}{c}\text { Frequency } \\
(\mathrm{Hz})\end{array}$ & $\begin{array}{c}\text { Phase } \\
(\mathrm{rad})\end{array}$ \\
\hline \multirow{4}{*}{$\begin{array}{l}\text { The method } \\
\text { based on the } \\
\text { jitter } \\
\text { displacement } \\
\text { estimation } \\
\text { model } \\
\end{array}$} & \multirow{2}{*}{$\begin{array}{l}\mathrm{B} 1- \\
\mathrm{B} 2\end{array}$} & detected & 0.9911 & 0.6567 & -0.1374 \\
\hline & & error & 0.0840 & 0.0006 & 0.0267 \\
\hline & \multirow{2}{*}{$\begin{array}{l}\text { B2- } \\
\text { B3 }\end{array}$} & detected & 0.9096 & 0.6566 & -0.1310 \\
\hline & & error & 0.0025 & 0.0005 & 0.0203 \\
\hline \multirow{4}{*}{$\begin{array}{l}\text { The presented } \\
\text { method based } \\
\text { on the } \\
\text { integration } \\
\text { imaging } \\
\text { model }\end{array}$} & \multirow{2}{*}{$\begin{array}{l}\mathrm{B} 1- \\
\mathrm{B} 2\end{array}$} & detected & 0.9662 & 0.6567 & -0.1173 \\
\hline & & error & 0.0591 & 0.0006 & 0.0066 \\
\hline & \multirow{2}{*}{$\begin{array}{l}\text { B2- } \\
\text { B3 }\end{array}$} & detected & 0.9095 & 0.6566 & -0.1177 \\
\hline & & error & 0.0024 & 0.0005 & 0.0070 \\
\hline
\end{tabular}
process, so as to make the transformation from disparity curve to jitter curve more accurate. The results demonstrate the performance of the presented method in jitter detection.

Table 6. Detected results of the jitter curve

\section{CONCLUSIONS}

Satellite jitter widely exists in many satellites and affects their imaging process. Jitter detection is the precondition of the compensation for satellite jitter. This paper has proposed a jitter detection method based on the integration imaging model. The main contribution of the presented method is the construction of the integration imaging model, which achieves the transformation from disparity curve to jitter curve, as well as from image space to object space, according to the detailed imaging analysis. Experiments were conducted to evaluate the performance of the presented method. And the results have demonstrated that the application of the integration imaging model increased the jitter detection accuracy with the error of obtained amplitude, frequency and phase not more than 0.0591 px, $0.0006 \mathrm{~Hz}$ and $0.007 \mathrm{rad}$, respectively. Other advanced researches about image matching may further provide reference for the step of pixel-to-pixel matching in the presented method. Moreover, the future work will focus on applying the presented method to panchromatic images.

\section{ACKNOWLEDGEMENTS}

This work was supported by the National Key Research and Development Program of China (No. 2016YFB0501402) and the National Natural Science Foundation of China (No. 41801382). 


\section{REFERENCES}

Amberg, V., Dechoz, C., Bernard, L., Greslou, D., de Lussy, F., Lebegue, L., 2013. In-flight attitude perturbances estimation: Application to PLEIADES-HR satellites. Proc. SPIE, 8866, 206-209.

Ayoub, F., Leprince, S., Binet, R., Lewis, K.W., Aharonson, O., Avouac, J.P., 2008. Influence of Camera Distortions on Satellite Image Registration and Change Detection Applications. In Proceedings of the 2008 IEEE International Geoscience and Remote Sensing Symposium, Boston, MA, USA.

Barker, J. L., Seiferth, J. C., 1996. Landsat Thematic Mapper Band-to-Band Registration. In IEEE Int. Geoscience and Remote Sensing Symp., Lincoln, Nebraska, USA.

Bethel, J., 1997. Least Squares Image Matching for CE604. Purdue University, West Lafayette, IN, USA.

Chamberlain S. G., Washkurak, W. D, 1990. High speed, low noise, fine resolution TDI CCD imagers. Proc. SPIE, 1242, 252-262.

Fan, C., Zhang, B., 2010. Analysis on the dynamic image quality of the TDICCD camera. In Int. Conf. OPEE., Wuhan, China, 62-64.

Gwinner, K.; Scholten, F.; Preusker, F.; Elgner, S.; Roatsch, T.; Spiegel, M.; Schmidt, R.; Oberst, J.; Jaumann, R.; Heipke, C. Topography of Mars from global mapping by HRSC highresolution digital terrain models and orthoimages: Characteristics and performance. Earth Planet. Sci. Lett. 2010, 294, $506-519$

Hadar, O., Fisher, M., Kopeika, N. S., 1992. Image resolution limits resulting from mechanical vibrations. Part III: numerical calculation of modulation transfer function. Opt. Eng., 31(3), 581-589.

Hochman, G., Yitzhaky, Y., Kopeika, N. S., Lauber, Y., Citroen, M., Stern, A., 2004. Restoration of images captured by a staggered time delay and integration camera in the presence of mechanical vibrations. Appl. Opt., 43(22), 4345-4354.

Hu, K., Zhang, Y., Liu, W., 2018. High-Frequency Jitter Detection by Registration Error Curve of High-Resolution Multi-Spectral Satellite Image. In 26th International Conference on Geoinformatics, IEEE, Kunming, China.

Iwasaki, A., 2011. Detection and estimation satellite attitude jitter using remote sensing imagery. In Advances in Spacecraft Technologies, InTech, Rijeka, Croatia.

Jiang, Y., Zhang, G., Tang, X., Li, D., Huang, W., 2014. Detection and Correction of Relative Attitude Errors for ZY102C. IEEE Trans. Geosci. Remote Sens., 52(12), 7674-7683.

Mattson, S., Robinson, M., McEwen, A., Bartels, A., BowmanCisneros, E., Li, R., Lawver, J., Tran, T., Paris, K., Lroc, T., 2010. Early Assessment of Spacecraft Jitter in LROC-NAC. In 41st Lunar and Planetary Institute Science Conf., Woodlands, Texas, USA.

Mumtaz, R., Palmer, P., 2013. Attitude Determination by Exploiting Geometric Distortions in Stereo Images of DMC
Camera. IEEE Transactions on Aerospace and Electronic Systems, 49(3), 1601-1625.

Pan, J., Che, C., Zhu, Y, Wang, M., 2017. Satellite jitter estimation and validation using parallax images. Sensors, 17(1), 83.

Potucková, M., 2004. Image matching and its applications in photogrammetry. Institut for Samfundsudvikling og Planlægning, Aalborg Universitet.

Sun, T., Long, H., Liu, B., Li, Y., 2015. Application of attitude jitter detection based on short-time asynchronous images and compensation methods for Chinese mapping satellite-1. Opt. Express, 23, 1395-1410.

Takaku, J., Tadono, T., 2010. High Resolution DSM Generation from ALOS Prism-processing Status and Inflfluence of Attitude Fluctuation. In Proceedings of the 2010 IEEE International Geoscience and Remote Sensing Symposium, Honolulu, HI, USA.

Tang, X., Xie, J., Wang, X., Jiang, W., 2014. High-Precision Attitude Post-Processing and Initial Verification for the ZY-3 Satellite. Remote Sens., 7(1), 111-134.

Teshima, Y., Iwasaki, A., 2008. Correction of Attitude Fluctuation of Terra Spacecraft Using ASTER/SWIR Imagery with Parallax Observation. IEEE Trans. Geosci. Remote Sens, 46, 222-227.

Tong, X., Li, L., Liu, S., Xu, Y., Ye, Z., Jin, Y., Wang, F., Xie, H., 2015a. Detection and estimation of ZY-3 three-line array image distortions caused by attitude oscillation. ISPRS J. Photogramm. Remote Sens., 101, 291-309.

Tong, X., Xu, Y., Ye, Z., Liu, S., Tang, X., Li, L., Xie, H., Xie, J., 2015b. Attitude Oscillation Detection of the ZY-3 Satellite by Using Multispectral Parallax Images. IEEE Trans. Geosci. Remote Sens., 53(6), 3522-3534.

Tong, X., Ye, Z., Xu, Y., Tang, X., Liu, S., Li, L., Xie, H., Wang, F., Li, T., Hong, Z., 2014. Framework of jitter detection and compensation for high resolution satellites. Remote Sens., 6(5), 3944-3964.

Wang, H., Yang, Z., Chen, Y., Quan, W., 2012. A study on the influence of the satellite attitude accuracy on TDICCD imaging. In Proc. IEEE ISICT, London, UK, 219-223.

Wang, M., Fan, C., Pan, J., Jin, S., Chang, X., 2017. Image jitter detection and compensation using a high-frequency angular displacement method for Yaogan-26 remote sensing satellite. ISPRS J. Photogramm. Remote Sens., 130, 32-43.

Wang, M., Zhu, Y., Jin, S., Pan, J., Zhu, Q., 2016. Correction of ZY-3 image distortion caused by satellite jitter via virtual steady reimaging using attitude data. ISPRS J. Photogramm. Remote Sens., 119, 108-123. .

Wong, H. S., Yao, Y. L., Schlig, E. S., 1992. TDI chargecoupled devices: Design and applications. IBM J. Res. Dev., 36(1), 83-106.

Zhu, Y., Wang, M., Cheng, Y., He, L., Xue, L., 2018. An Improved Jitter Detection Method Based on Parallax Observation of Multispectral Sensors for Gaofen-1 02/03/04 Satellites. Remote Sens., 11(1), 16. 